\title{
Utilization of Colour for Visual Representation of Nature, Form, Space and Time
}

\author{
Isaac Ossei Agyekumhene $^{1^{*}} \quad$ G. Y. Annum ${ }^{2} \quad$ Emmanuel Antwi $^{2}$ \\ 1.P. O. Box PC 28, Ashanti Region, Ghana \\ 2.Department of Painting and Sculpture, Faculty of Art, Kwame Nkrumah University of Science and Technology, \\ Ghana \\ * E-mail of the corresponding author: agyekumhene1@gmail.com
}

\begin{abstract}
In nature, everything has a form, occupies a space, and is susceptible to change in a matter of time. Nature, form, space and time could well be classified as theoretical and the problem of intangibility of the said terms is addressed with the application of colour to visually represent them through paintings. Through application of colour, this article pulls the said incorporeities from their abstract realm for visual perception. Methodology of Observation was applied to observe some scenes at the same time, but a particular scene or spot was studied at four different periods of the day namely; morning, afternoon, evening and night. Participatory methodology was utilized to capture the scenes with a camera and, through the application of colour, painted the captured scenes on canvasses. Analyses of the painted scenes were finally made to result in conceptually representing nature, form and space with painted objects. Four of the scenes visually present the four periods or time of the day which are morning, afternoon, evening and night. The analyses have subsequently resulted in visually bringing about the concept of nature, form, space and time. In conclusion, if the intangibilities of nature, form, space and time could be concretized for visual conception, then any theory could be practically materialized.
\end{abstract}

Keywords: colour, visual representation, nature, form, space, time

DOI: $10.7176 / \mathrm{ADS} / 94-01$

Publication date:July $31^{\text {st }} 2021$

\section{Introduction}

This article dwells largely on conceptual interpretations or analyses of paintings which, obviously, are executed from colour. Due to its immense significance, colour has received much attention from various artists both locally and internationally. Some of these artists utilise colour for aesthetic effects, psychological effects or purposes as well as colour symbolism and philosophical interpretation. This research, basically, philosophically concretizes the intangibility of Nature, Form, Space and Time with the application of colour. The scope of the research was limited to the Central Market which is a place where many different people and objects which represent colour simultaneously interplay. Subsequent to the fact that each object or scene owes its identity to colour, I represented colour with painted scenes from the Kumasi Central Market. Conversely, the scenes reproduced with colour render them stationary on specific surfaces or spaces, portraying the forms or objects, spaces and the time or the period of the day each was painted, for instance, morning, afternoon, evening or night, all of which could be classified under a period of time.

The paintings produced from colour are thus, used to, conceptually, concretize the ethereal terminologies of Nature, Form, Space and Time. Bits of elaborations are made on the said four subjects of the of the research but more light is thrown on colour by virtue of the fact that it is the main medium used to create visual symbolisms of the themes. To achieve the said aims, the following processes were employed as methodology.

\section{Methodology}

The primary source of materials for this article came from Kumasi Central Market and the KMA through taking of pictures of scenes and interviews. Structured interviews were conducted with the help of interview guides or questionnaires to gather information from the authorities of Kumasi Metropolitan Assembly, (KMA), and the users of Kumasi Central Market. The market was visited at the four major periods of the day which are 1 . Morning, 2. Afternoon, 3. Evening and 4. Night. In the morning it was detected that light from the sun was dull, as a result, it was difficult to distinguish one colour from the other at a distance and even at close range, the hues are not so clear. In the afternoon the colours were so bright exuding energy and vitality. at the evening, the colours were dimmed losing their lustre. At night, only dark silhouetted images could be observed. The captured scenes were painted to be analysed to symbolize time as seen from figure $7-10$. The secondary data was collected through the use of archives, libraries and the internet as well as published and unpublished literature. As empirical studies, the works of selected artists who work in the same direction as my research were reviewed. Some of such artists were interviewed as well.

The practical aspect of the research was conducted at my private studio in my house. Experimentative, and Participatory methods were employed to prepare the canvases and painted the scenes. Analytical method was 
employed to discourse on the results of the Studio Practice as shown with Figures 1 to 10 . The analyses were basically conceptual and were influenced mostly by colour which was used for the paintings to portray humans and objects to symbolize nature, forms, spaces as well as the effect of light on colour to determine the period in time. Colour is thus discussed but before that, its source, which is light, has to precede.

\section{Results and Discussion}

Paintings of scenes from the Kumasi Central Market was successfully analysed to establish the fact that everything derives its identity from colour, and without colour, hardly could anything be seen let alone being identified. As a result, colours from the scenes were effectively utilized to represent colour as a concept. Colour, thus represented by the painted scenes, were philosophically analysed, in the form of symbolisms, to bring out the intangible forms of Nature, Form, Space and Time to be 'clothed with colour' for visual observation. The corporeal nature of the said forms has thus been concretized to be perceived as a result of the application of colour. Research on colour brought about its source, definition, its history and origin, its characteristics, its types as well as the said terms of Nature, Form, Space and time. The source of colour which is light, thus precedes the discussions.

\subsection{The Concept of Light}

Light is defined as a form of energy that travels in a straight line at three hundred million $(300,000,000)$ metres per second. Until it is blocked by something in its way, it will continue to travel at that rate, (Mwangi Saka\& Williams, 1974, pp. 5-8). When such an action takes place, part or all the light may be reflected, absorbed or transmitted by the thing or object that obstructs the movement of the light. Natural light (whether from the sun, the moon or the stars) is white and is composed of seven colours of the rainbow (Mwangi, Saka \& Williams, 1974, p.12). The fact that light is described as white does not necessarily imply opaque white but rather a transparent state of white. I would prefer to term light colourless since all colours are seen through it in their purest states. Light is therefore transparent and could be termed as colourless. That is the more reason why the hues of colour are easily formed from the amount of light transmitted on an object to portray the kind of colour which is discussed below.

\subsection{Definition of Colour}

Culled from the World Book Encyclopaedia, the definition claims that, "Colour is what the eye sees when light strikes it. Everything in the universe has colour; example, green light is reflected to our eyes when we see grass. Plato defined colour as a particulate flame given off from every sort of the body. The colour particles differ in size. Plato asserts that if the colour particles which are different in size are equal to the component of the visual influence, they are imperceptible and the colour is called the "transparent" or the "diaphanous."

Aristotle sooner adopted this term which also claimed that large particles from these bodies contract the compound, yielding the sensation of black, while similar particles dilate it, producing white. (Tanaka, Weiskopf \& Pepper, 2001. p. 211). Colour as defined by Aristotle is "The limit of the transparent medium in a definitely bounded body." But since then various definitions have come about. (Guerlac 1986, p.6). Purity in colour, so says Nassau, is the amount of white light mixed with a hue. It could also be termed as saturation. The colours: red, yellow, and blue, known as primary colours, can be combined in varying proportions to produce all other colours. Primary colours combined in equal proportions produce secondary colours. Two colours that combine to form white light are said to be complementary (Guerlac, 1986, p.6).

Having thus defined colour, it suffices to briefly elaborate on what its history and origin are.

\subsection{Origin and History of Colour}

Colour obviously existed from time immemorial. Adam and Eve, the first seed of humankind, as well as their descendants, obviously knew the difference in one colour from another due to the fact Adam named all objects (Genesis 2:19-20). As said earlier, everything in nature is identifiable trough colour so Adam definitely knew what colour is and might have named it. The earliest Greek philosophers' ideas concerning light and colour were only fragmentary until extended treatment by Plato. "Extromission" or "Visual ray" theory is the first theory of vision. It held that the external objects which we see are the result of fire-like emanation from the eye (Guerlac, 1986. p. 3). The Pythagorean, Alcmeon of Croton (5th Century B.C.) who gave as evidence the flashiness of light and colour seen when the eye is struck, was also a supporter of Extromission Theory. This theory was held by Empedocles as evidenced by Aristotle quoting ten lines from

Empedocles poem "On Nature," which the theory was clearly invoked, (Guerlac, 1986, p. 5). The idea conveyed by Aristotelian theory which viewed colour to be the product of a mixture of white and black held as 'law' until the 'Experimentum Crusis" by Isaac Newton in 1666. Having defined colour and briefly dilated on its historical origin, its characteristics as well as its types, are also briefly distended below with the characteristics preceding the types. 


\subsection{Characteristics of Colour}

Colour, according to Kurt Nassau, is associated with the visible electromagnetic radiation, which stimulates the sensor cells of the eye. Red light has the longest wavelengths, while blue has the shortest, with other colours such as orange, yellow, and green in between. Light, continues Nassau, is not the only type of electromagnetic radiation. He asserts that light is only a small segment of the totality of the electromagnetic spectrum, but it is the one form that can be perceived with the eye. Colour abounds everywhere and whatever could be seen owes its visibility to colour. Apparently, it is colour that creates the identity of any man or object. Without colour, you may agree with me that nothing could be identified. Yet what causes colour to behave differently in relation to the intensity of light? Is there a special quality in colour which enables it to behave as it is? What causes colour to behave in like manner? How does colour relate to light? Why does colour behave differently in relation to proximity or distance from light?

The implication is that, though everything is composed with colour, it behaves differently in presence of light. Colour, when exposed to intense light, brightens up, but as soon as the light dims, the hue of the colour loses its lustre. This characteristic of colour could be observed on humans, plants, animals and objects being hosts of colour. It is therefore quite apparent that colour does not commonly move about by itself without being found on an object. Subsequently, to effectively analyse colour philosophically, the very icons or hosts of colour which are everything around, was utilized as mediums. Conversely, to visually represent any item in nature, and to effectively analyse any of it as a concept, be it tangible or corporeal, colour, which is categorized into two major types, is the only plausible medium the artist could make use of. The two major categories of colour are termed as additive and subtractive and briefly elaborated below.

\subsection{Types of Colour \\ a) Additive Colour}

Additive Colour is the result when coloured light is added to another different coloured light. It results in the production of another different colour from the two addends. (Thompson, 1995, p. 150).

On the same page, additive colour is expatiated with the following examples. For unlike coloured pigments, the rudiments of coloured light follow somehow different rules that could be said of as unpredictable. There are similarities in certain mixtures yet some results are achieved differently with coloured light as compared with coloured pigments. For instance:

(1) When rays of blue, red, or green (the additive primaries) are overlapped, the

(2) secondary colours are created.

(3) Where blue light overlaps red light, magenta is resulted or created.

(4) The result of red light overlapping green light is yellow.

(5) Cyan is the result of blue and green light overlapping.

(6) White light is produced when blue, green and red-light rays overlap. This indicates that white light could be created by the presence of all the hues of colour (Thompson, 1995, p. 150).

\section{b) Subtractive Colour}

In a beam of daylight all colours are present and therefore it would not have been easy to identify the colour of an object as all the beam strikes on it. All coloured objects possess certain physical properties known as colour quality or pigmentation that enables the object to absorb some colour hues and reflect others. A black board is seen as black because the board reflects only the black hues in the ray of light and absorbs the rest. The artist's pigments have these properties and therefore what is applied to the surface of an object gives it the same characteristics. The surface pigmentation could be altered with dyes, chemicals or stains but no matter how the pigmentation is altered, a new colour is created as the rest of the hues are absorbed by the surface of the object leaving what is perceived as its colour. The colour, thus reflected, is the subtractive colour but not the actual light rays or the additive colour that strikes with its beam of coloured light (Thompson, 1995, p. 151). While colour (which emerges from light), is the actual identity of the object, it could not be detected unless reflected in a kind of hue. Yet the shape of the art form defined by lines could be observed without much light. In most cases, even in pitch darkness, the texture of the surface could be felt with the hand for its effect. Yet without colour, as stated above, no object has an identity. As a result, it is colour that composes everything in nature and by extension, nature itself.

\subsection{Nature}

The Oxford University Press Dictionary defines Nature as;

1. phenomena of the physical world collectively, including plants, animals, and the landscape, as opposed to humans or human creations (a) The physical force regarded as causing and regulating these phenomena (b) Archaic- A living thing's vital functions or needs.

2. The basic or inherent features, qualities, or character of a person or thing (a) Inborn or hereditary characteristics as an influence on or determinant of personality. 
From the above definitions, it is obvious, or could be inferred, that whatever does not exist as a result of creation could be classified under nature. Besides that, there has been a norm that the accepted system of activities that goes on is recognized as normal and therefore natural, irrespective of creation.

\subsection{Form}

Form, according to The Oxford University Press Dictionary, is, "Visible shape or configuration. (a) Style, design, and arrangement in an artistic work as distinct from its content. The objects and humans displayed in the picture have their own special spaces occupied due to the peculiarity of each form or shape which renders it different from others. Some of the forms are larger in size, some are taller, smaller etc. One thing about the forms is that all objects including humans in the picture are easily identifiable by virtue of their forms and colour. An interesting thing about each form is that it is always seen as occupying a space of its own as part of the general space of the market enclave.

Outside the confinement of the Kumasi Central Market are even majority of the forms in nature. Everything in nature has a form which occupies space no matter how small and cannot claim to be independent of space. It is a fact that there is nothing without a form in nature. Without the particular form or shape of any human, animal, plant or object, the identity of that object is not possible. In spite of colour being the major element of identification, were the form of an animal to change to that of humans, hardly would that animal be classified as non-human, for instance, a dog, goat, cow etc. Similarly, if the form of any man changes to that of an animal, that person can no more be considered amongst humans. It is not only colour that could be affected by time, but form is also dependent on time for the maintenance of its shape. If time were to extend its period of visit and stay with any object in nature, hardly could that animal, plant or even man maintain the same shape or form for the length of period of the visitation. The implication is that nothing in nature can maintain the same shape or form for a long period of time without changing.

\subsection{Space}

According to the Concise Oxford Dictionary, a space is "A continuous area or expanse which is free or unoccupied. For instance (a) A gap between printed or written words or characters. (b) Pages in a newspaper, or time between broadcast programmes available for advertising (c) the freedom to and scope to live and develop as one wish.

1. An interval of time (often used to emphasize that the time is short) eg. both cars were stolen in the space of a few hours.

2. The dimensions of height, depth and width which all things exist and move

(a) Also, outer space. The physical universe beyond the earth's atmosphere (b) The near-vacuum extending between the planets and stars. etc.

With the said definitions, there could be observed two different forms of spaces displayed from each painting. The first is the surface on which the painting itself is executed which is the whole size of the primed canvass. The other is the empty areas in-between each of the objects or items painted on the canvass.

Besides the spaces exhibited with the paintings are other forms of spaces that are always around. Looking roundabout, all the vacuum around are spaces, but hardly are they classified as such, unless specially or specifically demarcated. One of the commonest types of space is that displayed purposefully for advertisement on billboards and on walls usually by the side of highways requesting prospective users to rent. Another specified form of space could be sometimes be observed being advertised as "Office Space For Rent." The commonest ones, probably, are the spaces demarcated on the sides of certain streets as parking lots for vehicles.

One obvious and interesting thing about the forms of spaces is that, they are made visible by the application of colour. Whether those advertisements are in the pictorial forms or texts, the medium usually employed is colour for the said space to be observed visually by the viewer. Looking closely at the picture, some objects could be seen as packed in such a manner that there is hardly a space in between the items (Figure 1). There are others sparsely arranged on the space or surface of the canvass, displaying gaps which are also other forms of spaces which could be located in-between the spaces of the objects displayed on the surface of the canvas. (Figure 3). Users of the market, some hawking with others stationary could be observed densely crowded at certain areas in a manner that makes any passage through so hectic and almost impossible if one has a luggage each in both arms (Figure 4). Yet other places have enough spaces to permit almost free movement (Figure 3). Stores and stalls as well have their own spaces they have occupied with other spaces for human traffic and others for tables and wares as seen in Figure 2. All the said kinds of spaces described above are visibly displayed with each painting. Spaces have thus been concretized for ocular perception on another space prepared in the form of canvas with limited spaces of $95 \mathrm{~cm}$ by $70 \mathrm{~cm}$ and others. As said earlier, though the whole said space on the canvas is covered by colour or primer, there are other forms of spaces on the canvas which are visually displayed in-between humans and objects that compose the scene. There are also other spaces portrayed or occupied when the canvases are displayed on walls, tables or panels. By occupying their respective spaces, other spaces are 
further used and others created with the intervals between each canvas. Besides the spaces that could be observed in between the displayed canvases, there is another vast space for viewers to occupy which, though not concretized by colour, yet could be visually observed due to the paintings from colour that are occupying certain spaces, leaving those areas empty for observation as spaces. Besides the spaces displayed with colour are other spaces, one example of which is the wide area or expanse of land or field.

\section{Time}

Time, as defined by The Free Dictionary (2021) is;

1. Duration, considered independently of any system of measurement or any employment of terms which designate limited portions thereof.

2. A particular period or part of duration, whether past, present, or future, a point or portion of duration; as, the time was, or has been; the time is, or will be.

It is also defined by The Oxford University Press Dictionary as;

The indefinite continued progress of existence and events in the past, present, and the future, as regarded as a whole.

1. A point of time as measured in hours and minutes past midnight or noon.

2. The favourable or appropriate moment to do something.

The use of colour to visually portray time cannot be limited only to the paintings of the four periods of the day. The commonest and widely accepted forms of time are usually what are displayed by watches and clocks. There are two types of time displayed by the said watches and clocks which are in the manual and digital forms. Both the manual or digital types cannot be visually displayed without colour. With the manual, it is colour that makes the figures which read the current time visible to the eye, whether embossed or engraved. The digital type is always in the form of bright light colour mostly in black or red. In spite of the artistic nature of the watches or clocks, it is only the time which is the reason of the watch or clock being purchased. If the system that displays the actual time malfunctions, the significance of the watch or clock is utterly reduced, and as such, the owner quickly has to see to its maintenance.

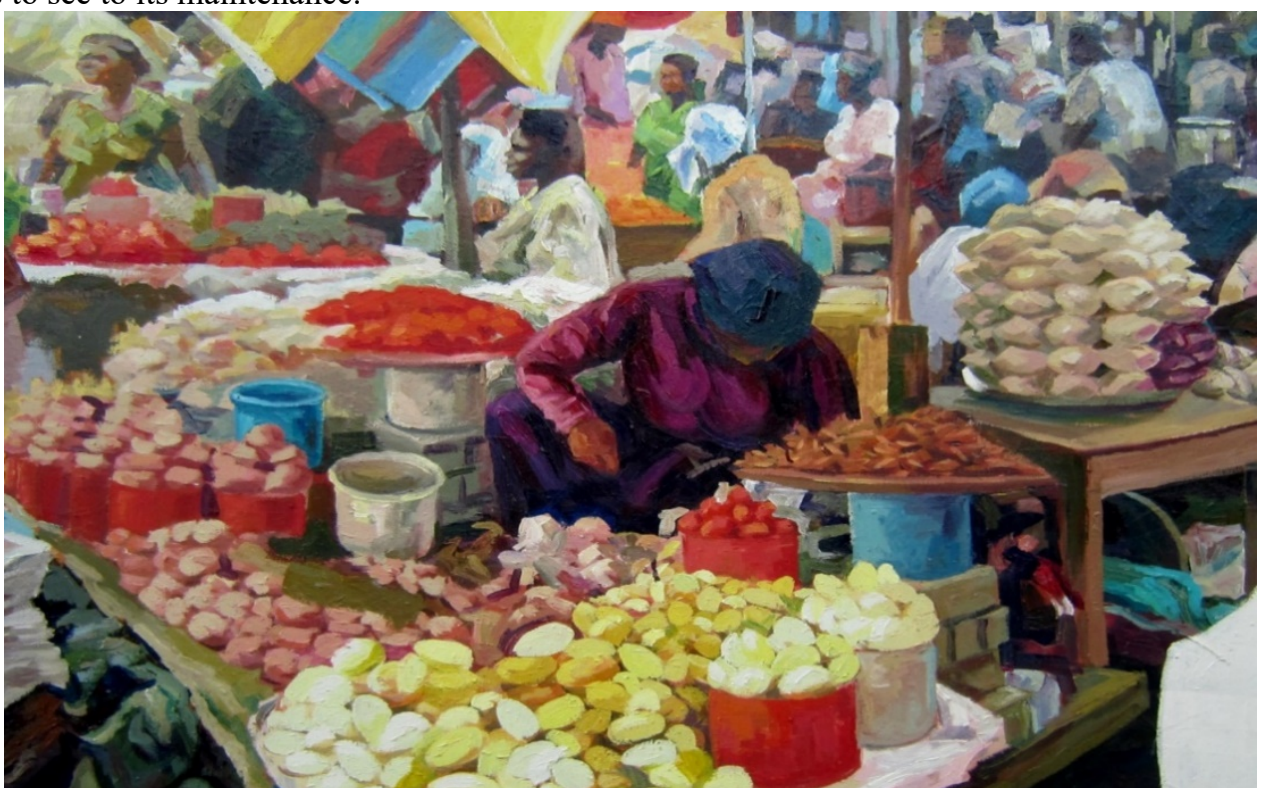

Figure 1: Lack of Space 


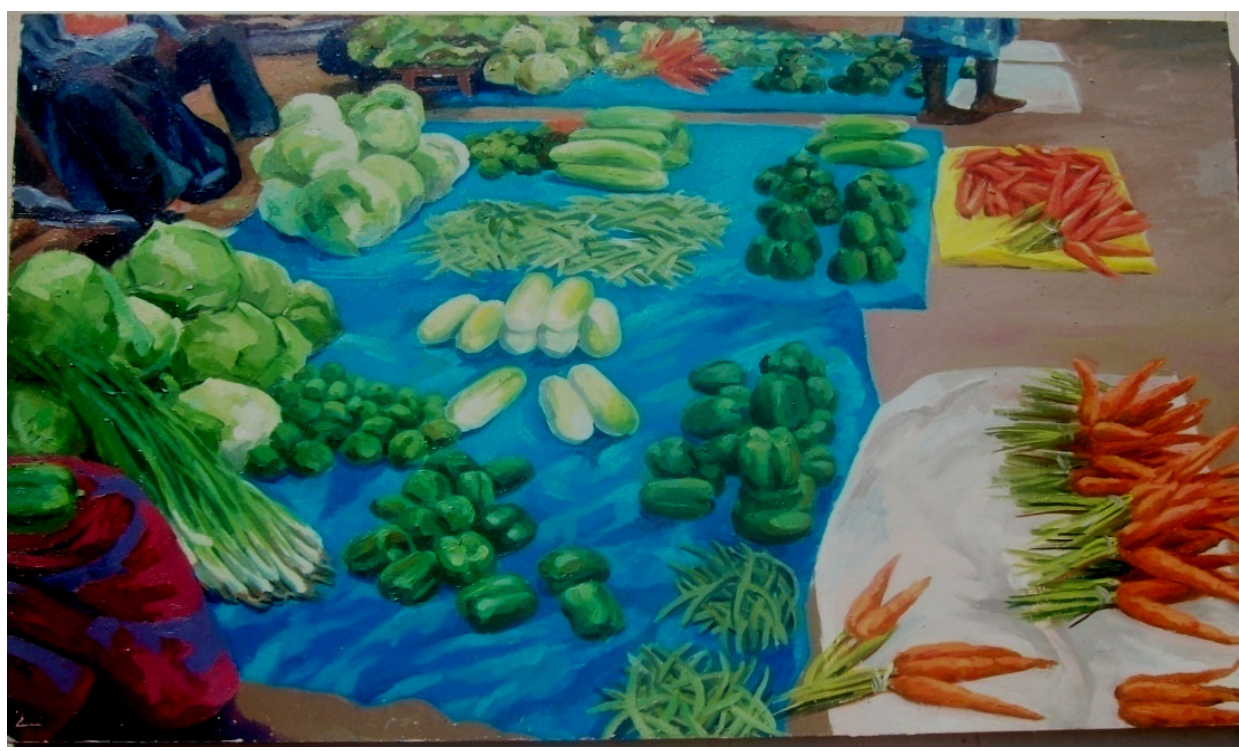

Figure 2. Spaces in-between wares

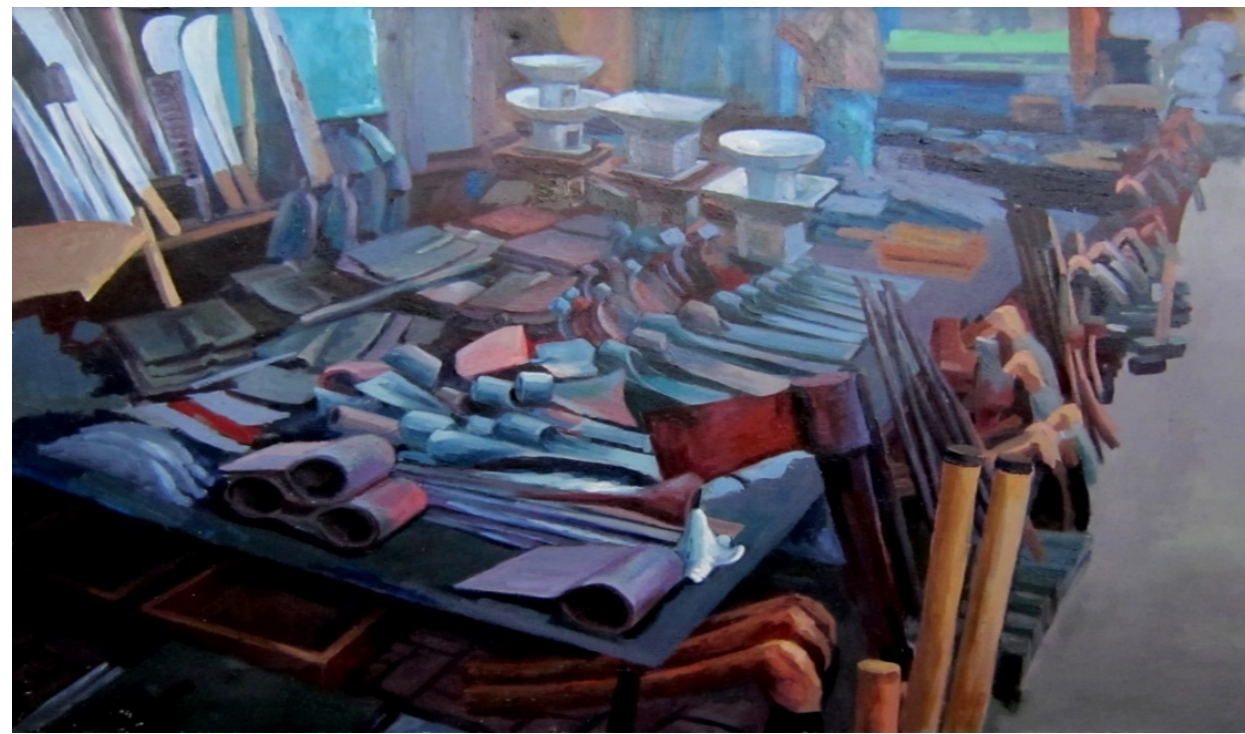

Figure 3. Spaces in Front of Stores for Table, Wares and Human Traffic

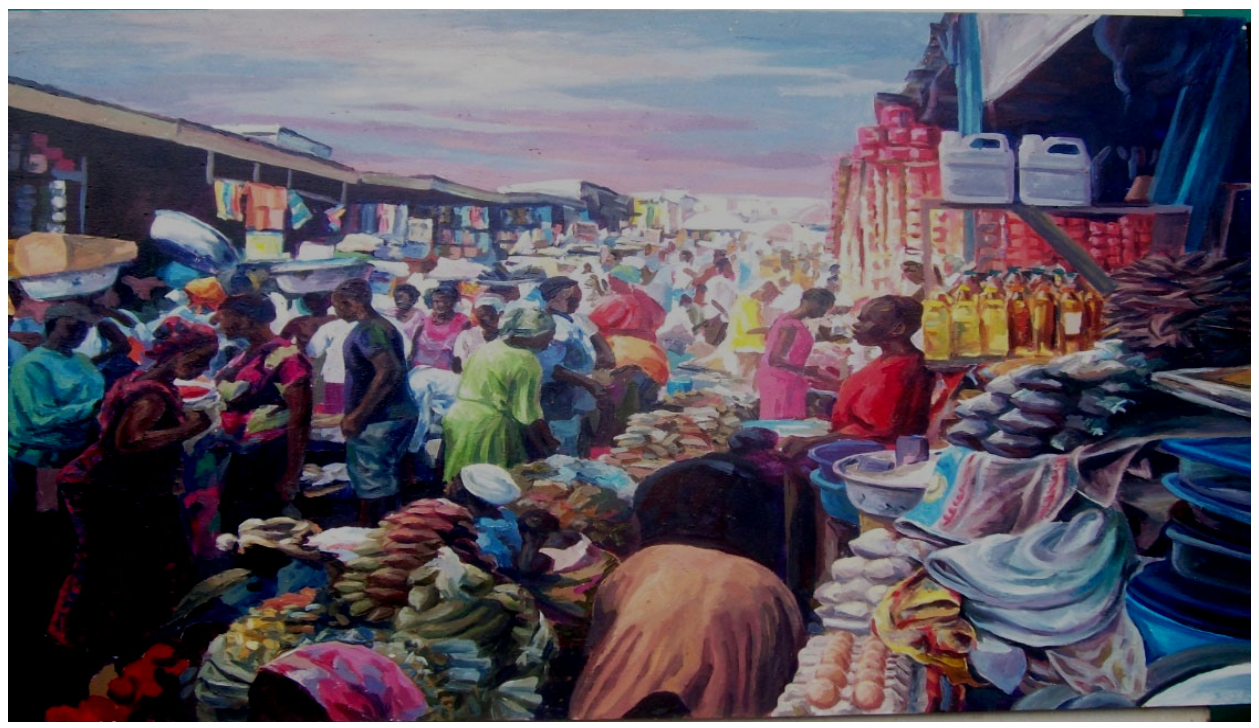

Figure 4. Congestion on space 
The activities of functioning and malfunctioning are also subject to time. With time the watch or clock could malfunction and with time, they could be restored to normalcy. An interesting concept is that time is subject to itself due to the fact that it could be affected by itself. At an expanse or a long period of time, the time of the clock or watch may not be reparable any longer and is thus annihilated. If time could adversely affect itself, then what else could be spared by it? Its grip could be felt at the throat of nature itself and therefore the whole universe. As said severally, all objects in nature that occupy space, and the space itself, are subservient to time. With time, changes affect all categories in nature. Nature itself which is composed of everything in the natural form, their shapes or forms, and even the spaces they occupy, could change in the course of time. The shape or form of every human or object changes with time. All forms of space could also be affected with time. For instance, the space of a football field could be filled to capacity within a short time and could be empty again in no time, after the close of the sporting activities.

Crops could be planted on a piece of land to fill almost all the space of the whole field. With time the crops would be harvested to render back the same empty space on the field again. Space of the crop field and the football field are not only affected by time but colour as well. The confetti of colours from the costumes of the players, officials and spectators make the space of the sports field visibly occupied. But with time, the stadium would be empty again with no colour seen and the former various forms of spacious spaces restored to their usual states or positions at the stadium. Similarly, is the field of crops affected by time and colour. The greenery or foliage of the crops that occupy the space of the field changes with time. With time the greens change to yellowish browns or other colours before they are eventually harvested to restore the space back to its former state on the field.

With time, spaces occupied by vehicles at the sides of some streets could be filled and could rapidly be emptied for other vehicles to later occupy. When colour from the demarcated portions with lines of white colour, and that of the vehicles make the spaces occupied, it is time that ensures that it is ripe for any vehicle to move away for others. at the end of the rented time one ought to leave, creating a space for others. The space on the canvas was empty before the painting, but with time, a scene was painted to fill the whole space of the canvass. Conversely, time, which is ethereal, and affects everything, could also be affected by colour. Apart from the effect of colour on time discussed above, time could be visually displayed with colour in the form of painting. A painted scene could portray the period or time of the day it was painted (Figures $6 \& 10$ ).

It is quite obvious that the paintings are night scenes even though one is not depicted by the title. Other scenes from my paintings as well portray the period of the day of each painting which are titled (a) Morning (b) Afternoon (c) Evening and (d) Night.

\section{Conclusion}

From the scope or the research population of the Kumasi Central Market, there have emerged sound premises for philosophical or conceptual inquiries into concretization of intangibilities. The Central Market has been the major source of the primary data and thus worth a bit of philosophical discourse like the major themes of the research. It is thus an arena of wealth, yet it is saddled with challenges some of which emerge in times of rains and fire outbreaks. The users, as well, are affected by the challenges in spite of the revenue generated on daily basis.

Such is the situation in life; there is no place without challenges or no one without a problem in life. There are usually some challenges but one has to learn endurance and the means of overcoming any challenge(s) that may rear up in life. The challenges off humans are as varied as they are numerous, in similar relation to the various colours. Such are the behavioural tendencies of humans which are as varied as colours are. Character is like colours, some are very pleasant or appealing, some neutral while others are quite repugnant, obnoxious or repulsive. Like hot, warm, neutral or cool colours, there are people with hot or cool tempers. There are also the proud or pompous with turgid or egocentric demeanour and those who are mild and respectful as well as others who live simple or modest lives and also the reserved and what have you. 


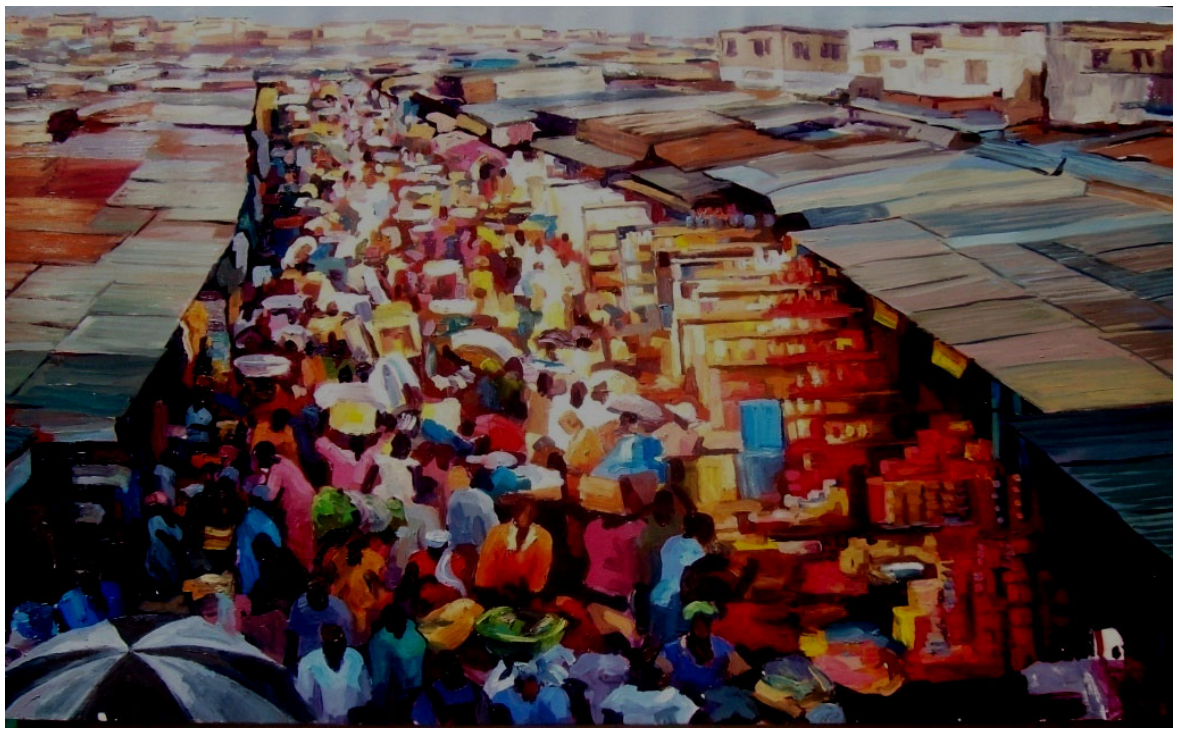

Figure 5. Evening Scene - The sun is setting behind the storeyed buildings casting shadows on the market

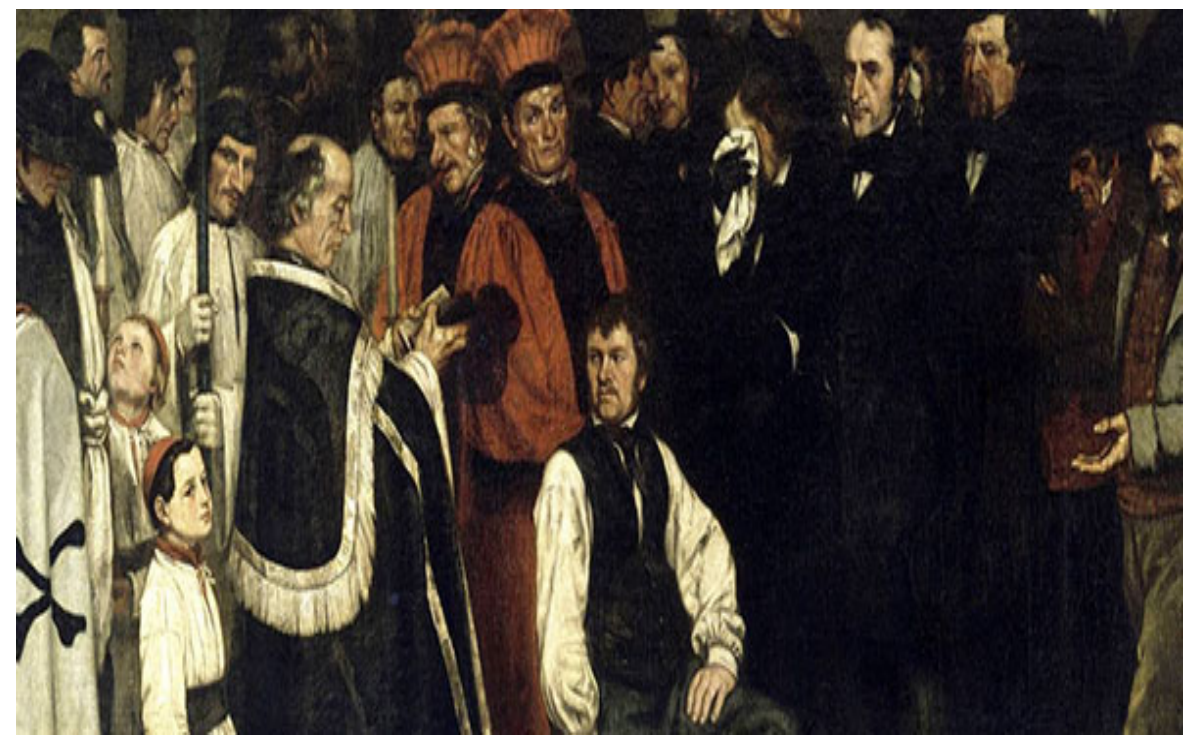

Figure 6. Burial at Ornans-(Figure 4). (Gustave Courbert -1849-51, the Launch of Realism)

https://www.theartstory.org/movement-realism.htm 


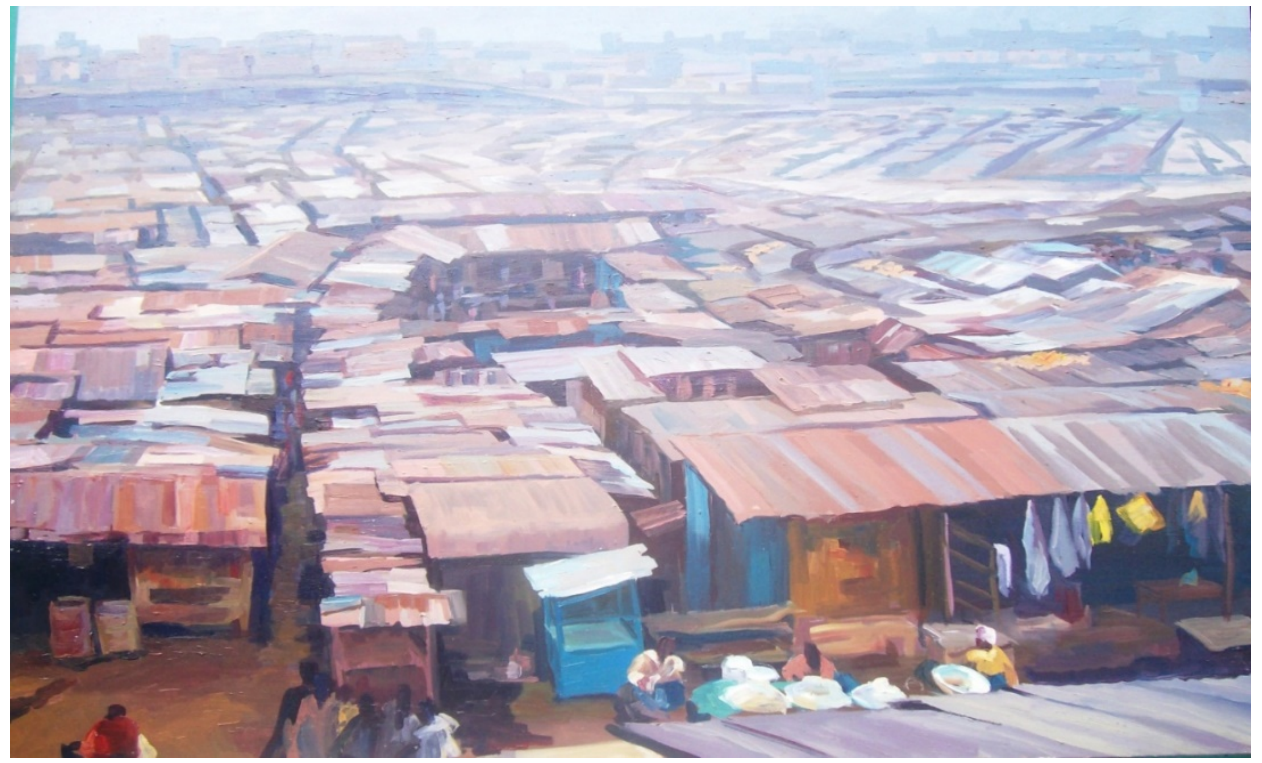

Figure 7. Morning

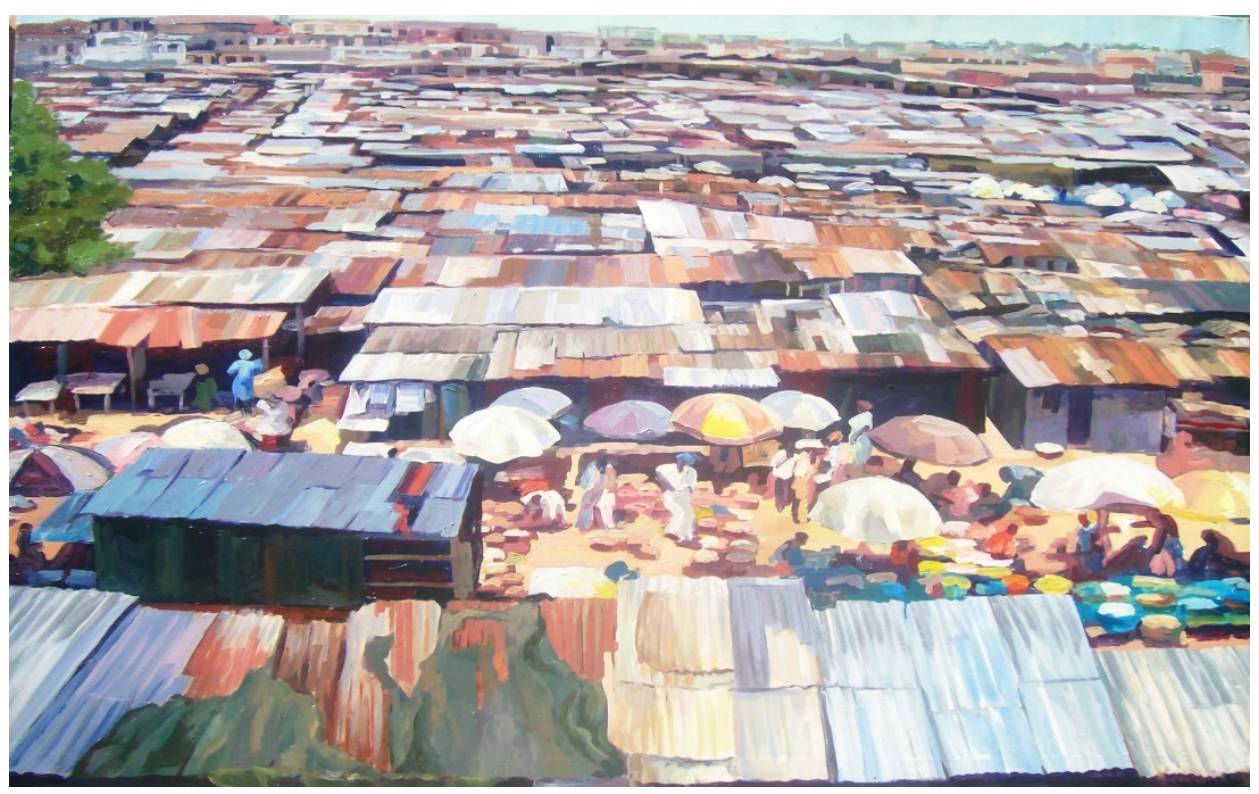

Figure 8. Afternoon 


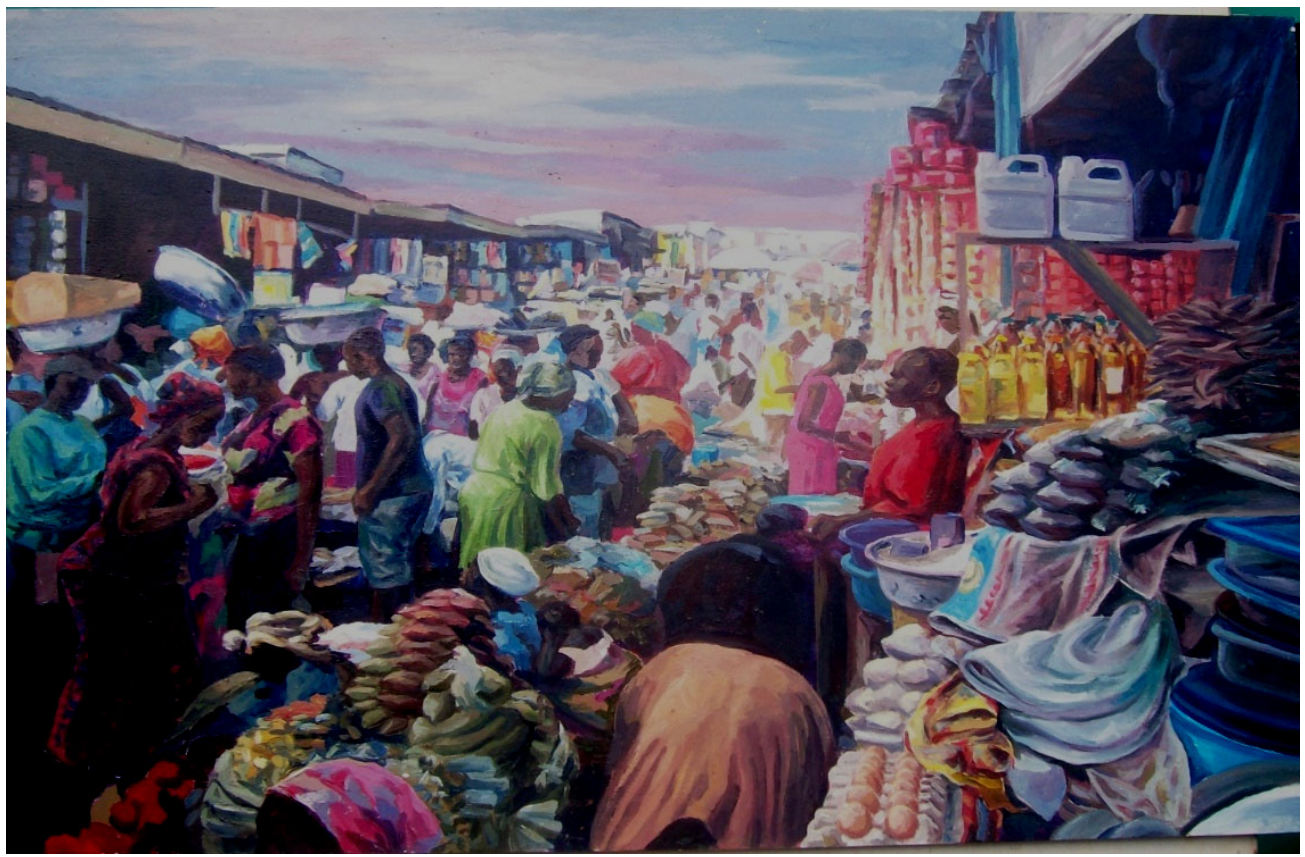

Figure 9. Evening

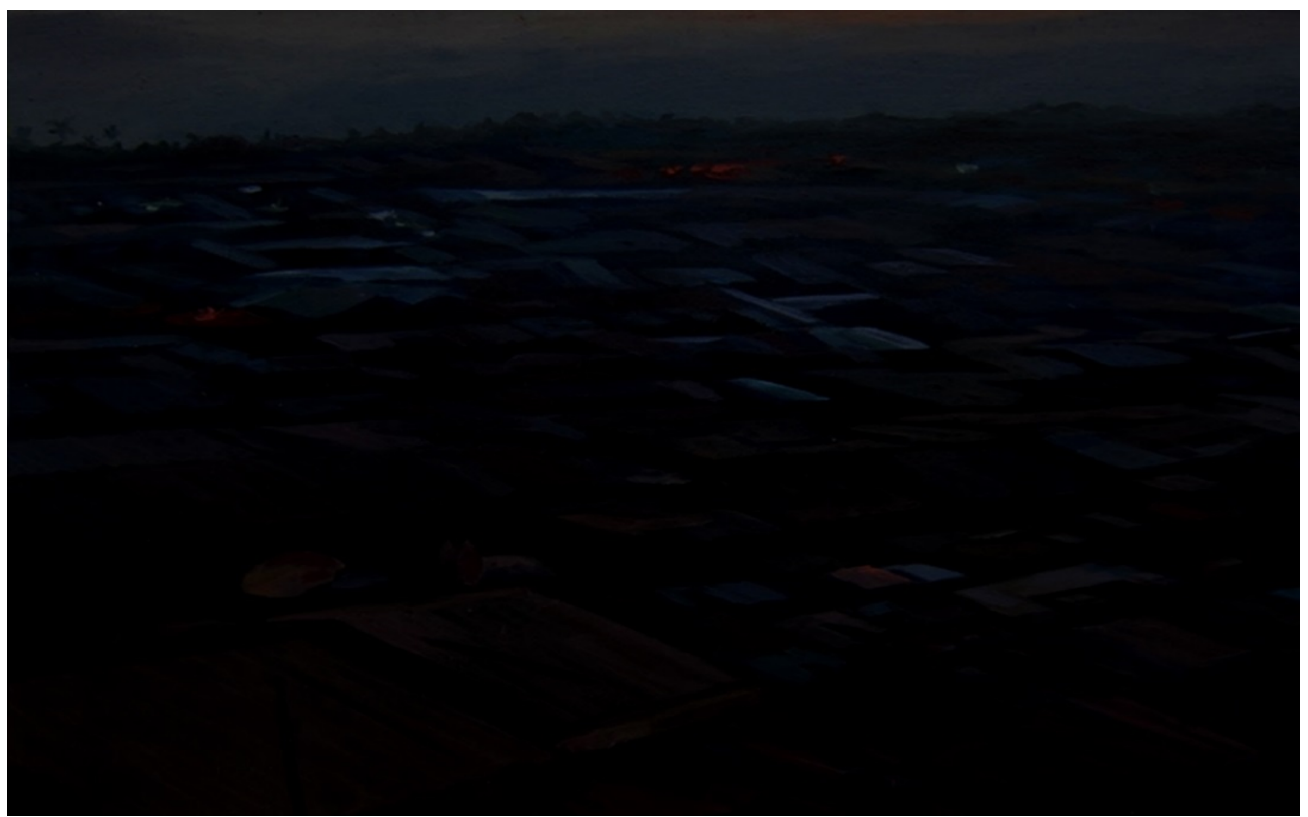

Figure 10. Night

Conceptually, society is consisted of people with diverse character traits, which, like colours or objects, could be differently observed, according to the distance between the object and the viewer. Like the hues of colour, the temperaments or characters of humans are best observed when living close to the one. The further one is from another, the more difficult it becomes being familiar with the one's life or character.

All the discussions and comparisons border on the premise that, in as much as there are difference in everything in nature, the forms, the sizes of space occupied as well as the time allotted for every activity and everyone, all the four theoretical terms and their symbolisms which are also intangible, have been made possible by the fact that nature, form, space and time have all been conceptually concretized by colour, through paintings of scenes from the Kumasi Central Market.

\section{References}

Concise Oxford Dictionary

Encyclopedia Britannica Online: http://www.britannica.com/EBchecked/topic/126658/colour.

Guerlac, H. (1986). Can There Be Colors In The Dark? USA. University of Pennsylvania Press.

Mwangi, M. Saka, E.C. \& Williams G.A. (1974). BASIC SCIENCE SERIES FOR AFRICA.BOOK 6.LIGHT. 
Hong Kong: McGraw-Hill Far Eastern Publishers (s) Ltd.

Newton 1671/1953. The New Theory about Light and Colour They published it in their Philosophical Transaction, on 19th February, 1671/2.

Newton 1730/1952) the first edition of which appeared in 1704, is cited in Thompson E. (1995) Wasserman, (1978), as cited in Thompson, (1995).

TheFreeDictionary.com (C) Farlex, Inc. 2021.

Otto, O., Robert, E.S., Philip, R., Robert, O. B., David, L. C. (2002). Art Fundamentals. Theory An Practice.School Of Art. Bowling Green University. New York. McGraw- Hill Companies.

Pepper Williams and James from Department of Psychology, Oberlin College, and Daniel Weiskopf, Department of Psychology, University of Massachusetts-Boston.

Tanaka, Weiskopf \& Pepper, 2001. p. 211).

TheFreeDictionary.com (C) Farlex, Inc. 2021.

The Oxford University Press Dictionary

World Book Encyclopaedia,

\section{References}

Arai, T., Aiyama, Y., Sugi, M. \& Ota, J. (2001), "Holonic Assembly System with Plug and Produce", Computers in Industry 46, Elsevier, 289-299.

Bell, G.A., Cooper, M.A., Kennedy, M. \& Warwick, J. (2000), “The Development of the Holon Planning and Costing Framework for Higher Education Management”, Technical Report, SBU-CISM-11-00, South Bank University, 103 Borough Road, London, SE1 0AA.

Bongaerts, L. (1998), "Integration of Scheduling and Control in Holonic Manufacturing Systems", PhD Thesis, PMA Division, K.U.Leuven.

Deen, S.M. (1993), “Cooperation Issues in Holonic Manufacturing Systems”, Proceedings of DIISM’93 Conference, 410-412.

Techawiboonwong, A., Yenradeea, P. \& Das, S. (2006). A Master Scheduling Model with Skilled and Unskilled Temporary Workers", Production Economics 103, Elsevier, 798-809.

Valckenaers, P., Van Brussel, H., Bongaerts, L. \& Wyns, J. (1997), "Holonic Manufacturing Systems", Integrated Computer Aided Engineering 4(3), 191-201.

Van Brussel, H., Wyns, J., Valckenaers, P., Bongaerts, L. \& Peters, P. (1998), "Reference Architecture for Holonic Manufacturing Systems: PROSA”, Computers in Industry 37(3), 255-274. 